\title{
SART1 Gene
}

National Cancer Institute

\section{Source}

National Cancer Institute. SART1 Gene. NCI Thesaurus. Code C129081.

This gene is involved in spliceosome activity. 